\title{
Concurrent or sequential use of cytotoxic chemotherapy and hormone treatment in advanced breast cancer: report of the Swiss Group for Clinical Cancer Research
}

\author{
F CAVALLI, M BEER, G MARTZ, W F JUNGI, P ALBERTO, J P OBRECHT, B MERMILLOD, \\ $\mathrm{K}$ W BRUNNER
}

\begin{abstract}
In a trial of combined hormone treatment and cytotoxic chemotherapy 464 patients with advanced breast cancer were randomly allocated to either concurrent or sequential treatment. Cytotoxic drugs were given only if the antitumour activity of the hormone treatment was inadequate. Hormone treatment consisted of oophorectomy for premenopausal and tamoxifen administration for postmenopausal patients. Length of survival was better, though not significantly, in premenopausal patients $(p=0.29)$ treated concurrently and in postmenopausal women $(p=0 \cdot 17)$ treated sequentially; the difference was highly significant $(p=0.003)$ only for postmenopausal women in the low-risk category. The
\end{abstract}

Ospedale San Giovanni, 6500 Bellinzona, Switzerland

F CAVALLI, MD, head of the division of oncology

$M$ BEER, MD, senior registrar, division of oncology

University Hospital, 8091 Zurich, Switzerland

G MARTZ, MD, professor, division of oncology

Kantonsspital, 9007 St Gallen, Switzerland

W F JUNGI, MD, consultant, division of oncology

Hôpital Cantonal, 1205 Geneva, Switzerland

P ALBERTO, MD, senior lecturer, division of oncology

Kantonsspital, 4004 Basle, Switzerland

J P OBRECHT, MD, professor, division of oncology

Operations Office, Schweizerische Arbeitsgruppe für Klinische

Krebsforschung, 1205 Geneva, Switzerland

B MERMILLOD, PHD, senior statistician

Inselspital, 3010 Berne, Switzerland

K W BRUNNER, MD, professor, Institute for Medical Oncology findings suggest that postmenopausal women with metastatic breast cancer should probably be treated primarily by carefully monitored hormone treatment.

\section{Introduction}

Breast cancer tissue is thought to be composed of at least two different cellular types, one which is responsive to hormone treatment and one which is sensitive to cytotoxic drugs. ${ }^{1}$ A combination of both treatments should therefore improve the results of treatment of advanced breast cancer, particularly as no appreciable progress seems to occur when only one of the currently available treatments is used. The first trials of the efficacy of combining hormone and cytotoxic drug treatment yielded conflicting results. ${ }^{2-4}$ Nevertheless there is a widespread tendency to treat many patients with a combination of both treatments simultaneously. In our randomised study we compared this approach with more conservative management, in which chemotherapy was given only if the antitumour activity of hormone treatment was insufficient.

Patients and methods

SELECTION

Between September 1975 and December 1980, 464 patients with a measurable advanced breast cancer who had not previously received chemotherapy or hormone treatment were entered into the trial. Patients with brain metastases and impaired renal or hepatic function were excluded. Osteoblastic boney metastases and malignant effusions were not accepted as measurable lesions. Fifty-eight patients had to be excluded from the present analysis, because in seven tumour size was difficult to evaluate, in 42 there was a major protocol violation, and in nine death occurred within four weeks of starting treatment. The patients were stratified according to menopausal status and risk category, based on retrospective analysis of two of our previous studies. ${ }^{5}$

Low-risk category-This was based on the following features: (a) 
contralateral nodal metastases only, with a free interval between mastectomy and diagnosis of the first metastasis of at least two years; (b) boney metastases only, irrespective of the free interval; and (c) no more than two of the following: (i) lung or liver metastases (not both) with a free interval of at least four years; (ii) an isolated boney metastasis with a free interval of at least two years; (iii) skin metastases or an ipsilateral malignant pleural effusion with a free interval of at least two years or both; and (iv) ipsilateral nodal metastases and a free interval of at least two years.

High-risk category-This included all the remaining patients. Hormone receptors were not considered, since their determination was generally possible only towards the end of the accrual period.

\section{RANDOMISATION}

After stratification patients were randomised to treatment groups A (concurrent hormone treatment and chemotherapy) or B (sequential hormone treatment and chemotherapy). Premenopausal women underwent oophorectomy and postmenopausal patients received 20 $\mathrm{mg}$ tamoxifen daily. In group $\mathrm{A}$ the patients received chemotherapy concurrently; in group B cytotoxic drugs were given only after 6-8 weeks of hormone treatment except in cases of confirmed tumour regression, in which chemotherapy was begun later and only after the tumour had been reassessed. At the time of randomisation to the two treatment groups, patients were also randomly allocated to three different regimens representing minimal, conventional, and intensive cytotoxic treatment. The details of these three regimens and the results of the comparison among the three groups, showing significant differences in response rates but similar survival curves, have been published elsewhere. ${ }^{6}$ The two randomisation procedures yielded an equal number of patients in groups $\mathrm{A}$ and $\mathrm{B}$ receiving each of the different cytotoxic regimens.

\section{EVALUATION OF TREATMENT RESPONSE}

The distribution of the major prognostic factors among patients treated with concurrent (group A) or sequential (group B) drug and hormone treatments is shown in table I. The non-evaluable cases (30

TABLE I-Major prognostic factors in patients receiving
hormone treatment and cytotoxic drugs either concurrently (group $A$ ) or sequentially (group $B$ )

\begin{tabular}{lcc}
\hline & \multicolumn{2}{c}{ Treatment group } \\
\cline { 2 - 3 } Characteristics & $\mathrm{A}$ & $\mathrm{B}$ \\
\hline Median age (years) & $57 \cdot 5$ & $58 \cdot 5$ \\
Median free interval (months) & $26 \cdot 5$ & $23 \cdot 5$ \\
Premenepausal $(0)(\mathrm{n}=109)$ & 27 & 27 \\
Postmenopausal $(\%)(\mathrm{n}=297)$ & 73 & 73 \\
High-risk category $(\%)(\mathrm{n}=306)$ & 25 & 24 \\
Low-risk category $(\%)(\mathrm{n}=100)$ & 75 & 76 \\
Mean No of metastatic lesions & $2 \cdot 13$ & $2 \cdot 15$ \\
Site of metastases $(\%)^{*}:$ & 8 & 9 \\
(a) Local & 17 & 18 \\
Skeletal & 3 & 2 \\
Local + pleura & 15 & 20 \\
Local + skeletal & 12 & 9 \\
Visceral + local & 33 & 31 \\
Visceral + skeletal & 12 & 11 \\
Visceral only & 18 & 12 \\
(b) Liver & 41 & 40 \\
Lung & 6 & 9 \\
Local + soft tissue & 31 & 39 \\
Bone & 3 & 1 \\
Others &
\end{tabular}

*All patients are accounted for in both groups $(a)$ and $(b)$.

in group A, 28 in group B) and the reasons for their exclusion from the analysis were evenly distributed. For the evaluation of the therapeutic result the following categories were used : partial remission, minor response, no change, and progressive disease. A partial remission corresponded to a decrease of more than $50 \%$ in the sum of the products of the two largest diameters of all measurable lesions. In the case of osteolytic metastases either a complete recalcification or a partial recalcification indicated by a decrease in size were required. A partial remission had to last at least two months. No change meant that tumour size remained unchanged or the variation was less than $25 \%$. A minor response was defined as clear cut tumour shrinkage, which did not reach the limits of a partial remission. The disease was considered to be progressive if new lesions appeared or the size of the measurable lesions increased by more than $25^{\prime \prime}$, ..

\section{STATISTICAL ANALYSIS}

The survival period and the time to progression of the disease were calculated from entry into the study. Actuarial curves were based on the method described by Kaplan and Meier. " The statistical comparison among actuarial curves was carried out using the log-rank test, the Mantel-Cox test, and the generalised Wilcoxon test. ${ }^{8}$ In the case of differences among the statistical tests the least significant result was recorded.

\section{Results}

Of the 406 evaluable patients, 207 were allocated to group $\mathrm{A}$ and 199 to group B. The response rate is summarised in table II. For group $\mathrm{B}$ the best results with either hormone treatment or cytotoxic drugs were recorded. Patients achieving a partial remission with both treatments were counted only once. Among the premenopausal patients $46 \%$ had a partial remission in group $\mathrm{A}$ and $43 \%$ in group $\mathrm{B}$. Among the postmenopausal women $40 \%$ had a partial remission in group A and $33 \%$ in group B. A minor response was seen more often in group B than in group A $(26 \%$ compared with $25 \%$ in the premenopausal and $28 \%$ compared with $22 \%$ in the postmenopausal women).

TABLE II-Overall response rate to concurrent (group $A$ ) or sequential (group B) hormone treatment and cytotoxic chemotherapy in premenopausal and postmenopausal women

\begin{tabular}{llcccc}
\hline $\begin{array}{c}\text { Menopausal } \\
\text { status }\end{array}$ & Treatment & $\begin{array}{c}\text { No of } \\
\text { patients }\end{array}$ & $\begin{array}{c}\text { Partial } \\
\text { remission } \\
(\%)\end{array}$ & $\begin{array}{c}\text { Minimal } \\
\text { response } \\
(\%)\end{array}$ & $\begin{array}{c}\text { Total } \\
(\%)\end{array}$ \\
\hline Premenopause & $\begin{array}{l}\text { Concurrent } \\
\text { Sequential }\end{array}$ & 55 & $25(46)$ & $14(25)$ & $39(71)$ \\
Postmenopause & $\begin{array}{l}54 \\
\text { Concurrent }\end{array}$ & 152 & $60(43)$ & $14(26)$ & $37(69)$ \\
& Sequential & 145 & $48(33)$ & $43(22)$ & $93(61)$ \\
& & & & & $89(61)$ \\
\hline
\end{tabular}

The therapeutic results of hormone treatment alone were evaluable only among the patients who received delayed chemotherapy (group B). After oophorectomy 12 of $54(22 \%)$ premenopausal patients had a partial remission. In a further eight $(15 \%)$ cases a minor response was seen. Among the 145 postmenopausal patients, treatment with tamoxifen produced a partial response in $25(17 \%)$ women but a minor response in $21(14 \%)$. The median time to disease progression after a partial remission or a minor response was 12.5 months in premenopausal women after oophorectomy and 10.7 months in postmenopausal women treated with tamoxifen. Forty-two per cent of those patients in group B who responded to hormone treatment had a second cytotoxic drug-induced tumour regression whereas $52 \%$ of the patients who did not respond to hormone treatment showed a remission with chemotherapy. The median time to disease progression again after a second cytotoxic drug-induced tumour regression was 12.5 months for patients responding to hormone treatment and 7.5 months for those showing disease progression with hormone treatment $(p=0.006)$. The median time to disease progression was similar for all three cytotoxic drug regimens : $19 \cdot 6$ months after a partial remission for patients receiving concurrent hormone treatment and chemotherapy (group A) and 12.5 months, from the start of chemotherapy, for the same result to be achieved with delayed chemotherapy (group B).

Figures 1 and 2 represent the survival curves for the premenopausal and postmenopausal patients in both groups. Among premenopausal patients the median survival time was 25.3 months for group $A$ and 21.0 months for group $B(p=0 \cdot 29)$. Among postmenopausal patients the median survival time was 23.7 months for group A and 27.5 months for group $B(p=0 \cdot 17)$. Figure 3 represents survival correlated with the overall therapeutic result; the median survival time was longer than 41 months for patients with a partial remission, less than seven months for those with progressive disease, and 23 and 21 months respectively for patients with a minor response and no change (partial remission vs minor response + no change vs progressive disease: $\mathrm{p}<0.0001$ ). 
The risk category, the free interval, and the localisation and number of metastatic sites had a statistically significant effect on survival. A multifactorial analysis correlating these prognostic factors with the two different therapeutic approaches failed to show statistically significant differences in most instances. The survival period tended to be consistently longer with concurrent chemotherapy in patients with a more aggressive disease (that is, a shorter free interval and less favourable pattern of metastatic lesions). Patients with a less aggressive disease, however (that is, longer free interval and more favourable metastatic sites) showed a similar not statistically significant tendency to live longer with sequential chemotherapy. Only in two subgroups was there a statistically significant difference in survival. Patients in the age group 40-45 years had a median survival time of $43 \cdot 1$ months in group $A$ and 18 months in group $B(p<0.05)$. Among the 80 post-

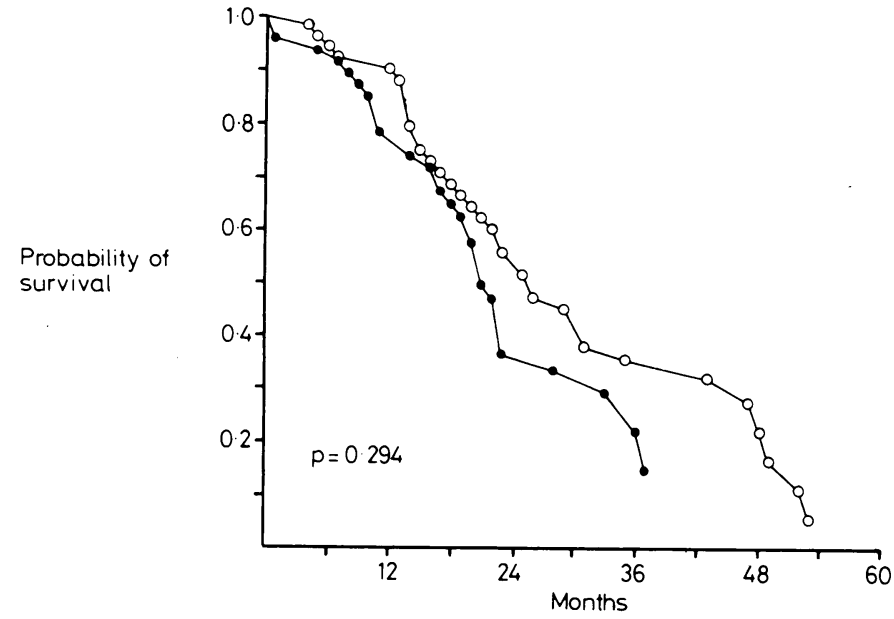

FIG 1-Survival curve for premenopausal patients receiving concurrent $(-)$ or sequential chemotherapy ( - ) after oophorectomy.

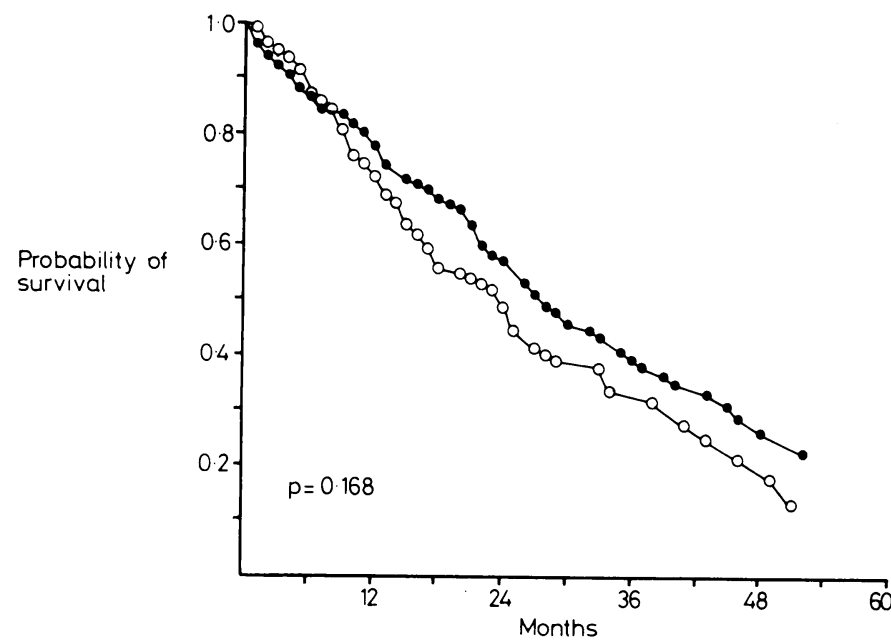

FIG 2-Survival curve for postmenopausal patients receiving tamoxifen and either concurrent $(-)$ or sequential chemotherapy

TABLE III-Median survival (in months) according to risk category and concurrent (group $A$ ) or sequential (group B) treatment with hormone and cytotoxic chemotherapy

\begin{tabular}{llll}
\hline \multirow{2}{*}{ Risk category } & \multicolumn{2}{c}{ Treatment group } & \\
\cline { 2 - 3 } & A & B & $\begin{array}{c}\text { Statistical } \\
\text { significance }\end{array}$ \\
\hline $\begin{array}{l}\text { Premenopausal women } \\
\text { Low-risk }\end{array}$ & $26 \cdot 6$ & 20 & NS \\
$\begin{array}{c}\text { High-risk } \\
\text { Postmenopausal women }\end{array}$ & $24 \cdot 2$ & $20 \cdot 9$ & NS \\
$\quad$ Low-risk & $21 \cdot 8$ & 40 & $\mathrm{p}=0.0034$ \\
& $23 \cdot 7$ & $22 \cdot 7$ & NS \\
\hline
\end{tabular}

NS - not significant

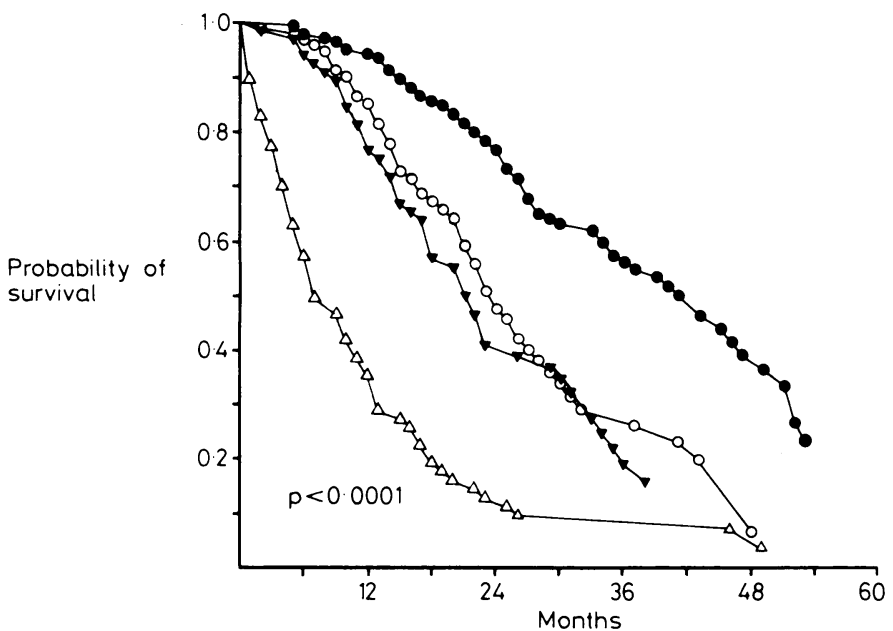

FIG 3-Survival curve of all patients in relation to therapeutic result : partial remission ( -0$)$, minor response $(\mathrm{O}-\mathrm{O})$, no change $(\boldsymbol{\Delta}-\boldsymbol{\Delta})$, progressive disease $(\triangle-\triangle)$

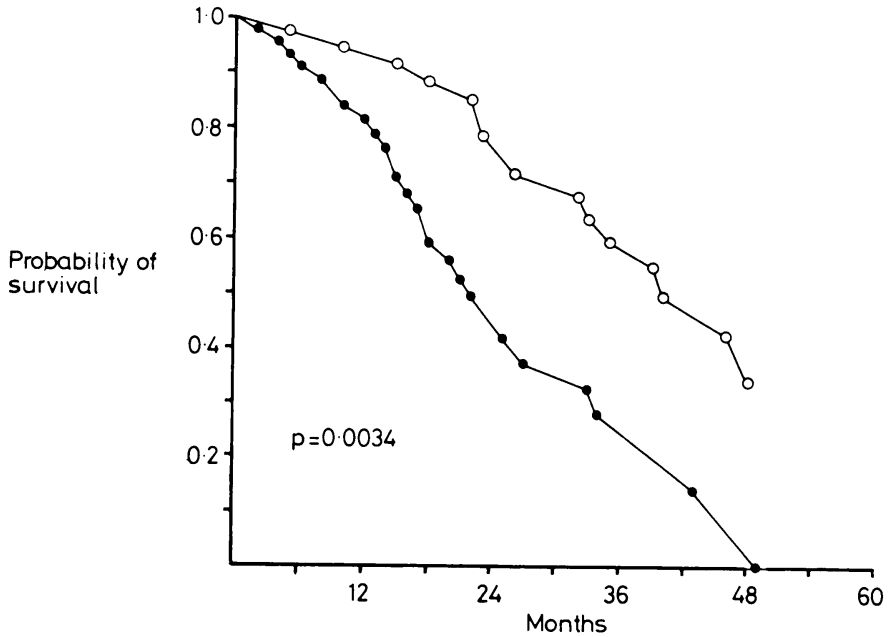

FIG 4-Survival curve for postmenopausal low-risk patients receiving tamoxifen and either concurrent (-O) or sequential chemotherapy $(c-0)$.

menopausal patients in the low-risk category, those receiving delayed chemotherapy had a median survival of 40 months and those receiving simultaneously tamoxifen and chemotherapy 21.8 months $(p=0.0034)$. These survival curves are represented in fig 4 . The median survivals according to menopausal status within the risk categories of the other subgroups of patients are summarised in table III.

\section{Discussion}

The best therapeutic approach to patients with metastatic breast cancer remains a matter of debate. It is well known that all effective hormone treatments will produce tumour regression in about one-third of patients. The response rate can only be increased if patients are selected according to one or more of the following criteria: content of hormone receptors of the tumour, well-defined favourable prognostic factors, and observed tumour regression due to previous hormone treatment. On the other hand the best regimens of cytotoxic drugs achieve tumour regression in $50-70 \%$ of patients, but only in $10-15 \%$ will all measurable lesions completely disappear; remissions are here generally more short-lived than with hormone treatment. ${ }^{9}$ The combined use of cytotoxic drugs and hormone treatment, the possibility of consolidating the achieved remission with radiotherapy (as in malignant lymphomas), and different very 
aggressive chemotherapeutic regimens are presently being explored to improve these results. ${ }^{10-12}$ At the same time, however, the overall impact of the use of multiple cytotoxic agents on the survival of most patients with advanced breast cancer has been questioned. ${ }^{13} 14$

Our results may partially support this assumption. In the analysis of the effect of cytotoxic drugs in our trial the statistically significant differences in the response rate with various cytotoxic regimens could only be marginally translated to the different survival curves. ${ }^{6}$ Differences in survival according to therapeutic result (fig 3) are of limited value in assessing the effect of treatment on survival. In fact some of the patients who responded might have survived almost as long without treatment, whereas in some patients who did not respond the treatment may have actually shortened their survival. At present probably only some subgroups of patients with advanced breast cancer will have their life significantly prolonged by chemotherapy.

Our trial is the third to assess the effects of simultaneous compared with sequential use of combined hormone treatment and chemotherapy in premenopausal patients with metastatic breast cancer. To our knowledge, however, such a study has so far not been carried out in postmenopausal women. Our results for premenopausal women confirm the findings of the two previous reports. ${ }^{15}{ }^{16}$ Overall, patients receiving cytotoxic drugs immediately after oophorectomy tended to live longer than patients for whom the onset of cytotoxic treatment was delayed; the difference, however, was not statistically significant. In all three trials a clear definition of the subgroups of premenopausal patients who would benefit from the concurrent use of both treatments has possibly been hampered by the difficulty in finding a sufficient number of suitable patients. Furthermore, the very similar survival data in our study for low-risk and highrisk cases brings into question the usefulness of this stratification in premenopausal women. Whereas in the two previous studies chemotherapy was given in cases in which the tumour had progressed after hormone treatment, in our trial cytotoxic drugs were also given to patients who showed no change with hormone treatment. The possibility that with a less prudent study design our differences in survival would have been more pronounced cannot be dismissed.

Among the postmenopausal patients women receiving cytotoxic drugs only after treatment with tamoxifen had failed to produce or maintain measurable tumour regression survived consistently longer, even if not statistically significantly, than those receving tamoxifen and cytotoxic drugs simultaneously. For patients with a less aggressive tumour, the low-risk group, the difference was highly significant (fig 3). Interestingly, the median survival was very similar with both treatments among postmenopausal patients in the high-risk category (table III). In the analysis of the different subgroups of postmenopausal patients according to their prognostic factors (that is, free interval and number and localisation of metastatic sites) we were unable to define a group which could have benefited from concurrent treatment with tamoxifen and cytotoxic drugs. Our data suggest that postmenopausal women with metastatic breast cancer should probably be primarily treated by a carefully monitored trial of hormone treatment and not initially with cytotoxic drugs, which in older patients can have unpleasant side effects. It is, perhaps, noteworthy that among the postmenopausal women the longest median survival was recorded for patients treated sequentially with the least intensive regimen of cytotoxic drugs. ${ }^{6}$

In our previous trial only a subgroup of patients receiving simultaneously cytotoxic drugs and oestrogens lived longer than patients treated with cytotoxic drugs alone. ${ }^{2}$ In a more recent and careful study of simultaneous treatment with cytoxan, methotrexate, fluorouracil, and tamoxifen in postmenopausal women compared with cytoxan, methotrexate, and fluorouracil alone, patients treated with the three drugs alone subsequently received the four-drug combination after primary or secondary failure of the combination without tamoxifen. ${ }^{17}$ Even if initial treatment with the four-drug combination produced a significantly higher response rate, survival was longer for patients treated initially with the three-drug combination and later by the same regimen supplemented with tamoxifen. This emphasises again the poor correlation between differences in the response rate to primary treatment and eventual survival of patients with breast cancer and supports, as do our present findings, a sequential therapeutic approach as superior in postmenopausal patients. Since subsequent remissions are possible with later hormone and cytotoxic regimens, more detailed clinical studies are necessary to define the most appropriate sequence of the various treatments available for postmenopausal patients with advanced breast cancer.

We are grateful to Professor W H Hartmann, LICR Berne Branch, for his critical review, and to O Kraitrova, for her careful preparation of the manuscript.

Correspondence should be addressed to Dr Franco Cavalli, Division of Oncology, Ospedale San Giovanni, 6500 Bellinzona, Switzerland.

\section{References}

${ }^{1}$ Henderson IC, Canellos GP. Cancer of the breast. The past decade. $N$ Engl 7 Med 1980;302:17-30,78-90.

Brunner KW, Sonntag RW, Alberto P, et al. Combined chemo- and hormonal therapy in advanced breast cancer. Cancer $1977 ; 39 \cdot 2923-33$.

${ }^{3}$ Carter SK. The interpretation of trials: combined hormonal therapy and chemotherapy in disseminated breast cancer. Breast Cancer Research and Treatment $1981 ; 1: 43-52$.

1 Tormey DC, Falkson G, Crowley J, Falkson HC, Voelkel J, Davis TE. Dibromodulcitol and adriamycin tamoxifen in advanced breast cancer. Am 7 Clin Oncol 1982;5:33-9.

${ }^{5}$ Fey MF, Brunner KW, Sonntag RW. Prognostic factors in metastatic breast cancer. Am $\mathcal{F}$ Clin Oncol 1981;4:237-48.

' Cavalli F, Beer M, Martz G, et al. Gleichzeitige oder sequentielle Hormono/Chemotherapie sowie Vergleich verschiedener Polychemotherapien in der Behandlung des metastasierenden Mammakarzinoms. Schweiz Med Wochenschr 1982;112:774-83.

' Kaplan EL, Meier P. Nonparametric estimation from incomplete observations. F Am Stat Assoc 1958;53:457-81.

' Peto R, Pike MC, Armitage P, et al. Design and analysis of randomized clinical trials requiring prolonged observation of each patient. II Analysis and examples. Br f Cancer 1977;35:1-39.

${ }^{9}$ Legha S, Buzdar AU, Smith TL, et al. Complete remissions in metastatic breast cancer treated with combination drug therapy. Ann Intern Med 1979;91:847-52.

1" Buzdar A, Blumenschein G, Montague E, Powell K, Hortobagyi G, Yap $H$. Regional consolidative therapy following systemic chemotherapy in metastatic breast cancer. Proc Am Soc Clin Oncol 1982;1:74.

"Tormey DC, Kline J, Davis TE, et al. Short term intensive chemohormonotherapy in metastatic breast cancer. Proceedings of the American Association for Cancer Research and the American Society of Clinical Oncology $1981 ; 22: 445$.

12. Vogel C, Lefante J, East D, et al. Cyclophosphamide, adriamycin and 5-fluorouracil alternating with a cycle-active regimen in metastatic breast cancer. A randomized Southeastern Cancer Study Group trial. Proceedings of the American Association for Cancer Research and the American Society of Clinical Oncology $1981 ; 22: 439$.

${ }_{13}$ Powles TJ, Coombes RC, Smith IE, et al. Failure of chemotherapy to prolong survival in a group of patients with metastatic breast cancer. Lancet 1980;i:580-1.

14 Paterson AHG, Szafran O, Cornish F, Lees AW, Hanson J. Effect of chemotherapy on survival in metastatic breast cancer. Breast Cancer Research and Treatment 1981;1:357-63.

15. Ahmann DL, O'Connell JO, Haan RG, et al. An evaluation of early or delayed adjuvant chemotherapy in premenopausal patients with advanced breast cancer undergoing oophorectomy. $N$ Engl $\mathcal{F}$ Med $1977 ; 297: 356-60$.

${ }^{16}$ Falkson G, Falkson HC, Glidewell O, Weinberg V, Leone L, Holland JF. Improved remission rates and remission duration in young women with metastatic breast cancer following combined oophorectomy and chemotherapy. A study by Cancer and Leukemia Group B. Cancer 1979;43: 2215-22.

17 Cocconi G, De Lisi V, Boni C, Mori P. CMF vs CMF plus tamoxifen (T) in postmenopausal metastatic breast cancer. A prospective randomized study. Proc Am Soc Clin Oncol 1982;1:75.

(Accepted 29 September 1982) 